\title{
On frequency-weighted coprime factorization based controller reduction
}

\author{
Andras Varga \\ German Aerospace Center \\ DLR - Oberpfaffenhofen \\ Institute of Robotics and System Dynamics \\ D-82234 Wessling, Germany \\ Andras.Varga@dlr.de
}

\begin{abstract}
We consider the efficient solution of a class of coprime factorization based controller approximation problems by using frequency-weighted balancing related model reduction approaches. It is shown that for some special stability enforcing frequency-weights, the computation of the frequency-weighted controllability and observability grammians can be done by solving reduced order Lyapunov equations. The new approach can be used in conjunction with accuracy enhancing square-root and balancing-free techniques developed for the balancing related coprime factors based model reduction.
\end{abstract}

\section{Introduction}

Controller synthesis methodologies like the LQG-, $\mathcal{H}_{2^{-}}$ or $\mathcal{H}_{\infty}$-design approaches (see for example [13]) lead often to controllers whose orders are too large for practical use. Therefore, in such cases it is necessary to perform controller reduction by determining a lower order approximation of the original controller. Controller reduction problems are often formulated as special frequency-weighted model reduction (FWMR) problems, where the frequency-weights are chosen to enforce closed-loop stability and an acceptable performance degradation when the low order controller is used instead the original high order one [1]. Interestingly, many stability/performance preserving controller reduction problems have very special structures which can be exploited when developing efficient numerical algorithms for controller reduction. For example, it has been shown in [11] that for the frequencyweighted balancing related approaches applied to several controller reduction problems with the special stability/performance enforcing weights proposed in [1], the computation of grammians can be done by solving reduced order Lyapunov equations.
The idea to apply frequency-weighted balancing techniques to reduce the stable coprime factors of the controller has been discussed in several papers [1, 5, 12]. While the frequency-weights in $[1,5]$ have been primarily chosen to guarantee closed-loop stability, it was shown in [12] that under mild conditions, performance bounds can be derived for coprime factors controller reduction in the case of a state-feedback and observerbased controller.

In this paper, we address the efficient solution of frequency-weighted balancing-related coprime factor controller reduction problems for the special stability preserving frequency-weights proposed in [5]. We show that for both general controllers as well as for statefeedback and observer-based controllers, the computation of frequency-weighted grammians for the coprime factor controller reduction can be done efficiently by solving lower order Lyapunov equations. Further, we show that these factors can be directly obtained in Cholesky factored forms allowing the application of the balancing-free square-root accuracy enhancing method for coprime factor reduction [10].

Notation. Throughout the paper, the following notational convention is used. The bold-notation $\mathbf{G}$ is used to denote a state-space system $\mathbf{G}:=(A, B, C, D)$ with the transfer-function matrix (TFM)

$$
G(\lambda)=C(\lambda I-A)^{-1} B+D:=\left[\begin{array}{l|l}
A & B \\
\hline C & D
\end{array}\right] .
$$

According to the system type, $\lambda$ is either the complex variable $s$ appearing in the Laplace transform in the case of a continuous-time system or the variable $z$ appearing in the $Z$-transform in the case of a discrete-time system. Throughout the paper we denote $G(\lambda)$ simply as $G$, when the system type is not indicated. The boldnotation is used consistently to denote systems corresponding to particular TFMs: $\mathbf{G}_{1} \mathbf{G}_{2}$ denotes the series coupling of two systems having the TFM $G_{1}(\lambda) G_{2}(\lambda)$, $\mathbf{G}_{1}+\mathbf{G}_{2}$ represents the (additive) parallel coupling of 
two systems with TFM $G_{1}(\lambda)+G_{2}(\lambda), \mathbf{G}^{-\mathbf{1}}$ represents the inverse systems with TFM $G^{-1},\left[\begin{array}{ll}\mathbf{G}_{1} & \mathbf{G}_{2}\end{array}\right]$ represents the realization of the compound TFM with $\left[G_{1} G_{2}\right]$, etc.

\section{Coprime factor controller reduction}

Consider $\mathbf{G}:=(A, B, C, D)$, an $n$-th order state-space model and let $\mathbf{K}$ be a stabilizing controller with a stabilizable and detectable $n_{c}$-th order state space realization $\mathbf{K}:=\left(A_{c}, B_{c}, C_{c}, D_{c}\right)$. The solutions of the frequency-weighted coprime factor controller reduction problems formulated in $[1,5]$ consist in computing approximations of the coprime factors of the controller. Specifically, the Frequency-Weighted Left Coprime Factor Reduction (FWLCFR) Problem is: given a left coprime factorization (LCF) $K=\widetilde{V}^{-1} \widetilde{U}$ of the controller, find $\mathbf{K}_{\mathbf{r}}$, an $r_{c}$-th order approximation of $\mathbf{K}$, in a LCF form $K_{r}=\widetilde{V}_{r}^{-1} \widetilde{U}_{r}$, such that the weighted approximation error

$$
\left\|\widetilde{W}_{o}\left[\widetilde{U}-\widetilde{U}_{r} \widetilde{V}-\widetilde{V}_{r}\right] \widetilde{W}_{i}\right\|_{\infty},
$$

is minimized. Similarly, the Frequency-Weighted Right Coprime Factor Reduction (FWRCFR) Problem is: given a right coprime factorization (RCF) $K=U V^{-1}$ of the controller, find $\mathbf{K}_{\mathbf{r}}$, an $r_{c}$-th order approximation of $\mathbf{K}$, in the RCF form $K_{r}=U_{r} V_{r}^{-1}$, such that the weighted approximation error

$$
\left\|W_{o}\left[\begin{array}{c}
U-U_{r} \\
V-V_{r}
\end{array}\right] W_{i}\right\|_{\infty}
$$

is minimized. In (1) and (2), $W_{o}$ and $W_{i}$ are stable weighting TFMs, which are specially chosen to enforce closed-loop stability.

Balancing related FWMR techniques which attempt to minimize (1) or (2) can be used to determine reduced order controllers. The following procedure to solve the FWLCFR Problem is based on the FWMR approach proposed by Enns in [2]:

\section{FWLCFR Procedure.}

1. Compute the controllability grammian of $[\widetilde{\mathbf{U}} \widetilde{\mathbf{V}}] \widetilde{\mathbf{W}}_{\mathbf{i}}$ and the observability grammian of $\widetilde{\mathbf{W}}_{\mathbf{o}}\left[\begin{array}{ll}\widetilde{\mathbf{U}} & \tilde{\mathbf{V}}\end{array}\right]$ and define according to [2], appropriate $n_{c}$ order frequency-weighted controllability and observability grammians $P_{E}$ and $Q_{E}$, respectively.

2. Using $P_{E}$ and $Q_{E}$ in place of standard grammians of $[\tilde{\mathbf{U}} \widetilde{\mathbf{V}}]$, determine a reduced order approximation $\left[\widetilde{\mathbf{U}}_{\mathbf{r}} \widetilde{\mathbf{V}}_{\mathbf{r}}\right]$ by applying, for example, the balanced truncation (BT) method [6] or the singular perturbation approximation (SPA) [4].

3. Form $\mathbf{K}_{\mathbf{r}}=\widetilde{\mathbf{V}}_{\mathbf{r}}^{-1} \widetilde{\mathbf{U}}_{\mathbf{r}}$.

A completely similar procedure can be used to solve the FWRCFR Problem:

\section{FWRCFR Procedure.}

1. Compute the controllability and observability grammians of $\left[\begin{array}{l}\mathbf{U} \\ \mathbf{V}\end{array}\right] \mathbf{W}_{\mathbf{i}}$ and $\mathbf{W}_{\mathbf{o}}\left[\begin{array}{l}\mathbf{U} \\ \mathbf{V}\end{array}\right]$, respectively, and define according to [2], appropriate $n_{c}$ order frequency-weighted controllability and observability grammians $P_{E}$ and $Q_{E}$, respectively.

2. Using $P_{E}$ and $Q_{E}$ in place of standard grammians of $\left[\begin{array}{l}\mathbf{U} \\ \mathbf{V}\end{array}\right]$, determine $\left[\begin{array}{l}\mathbf{U}_{\mathbf{r}} \\ \mathbf{V}_{\mathbf{r}}\end{array}\right]$, a reduced order approximation, by applying either the BT method [6] or the SPA [4].

3. Form $\mathbf{K}_{\mathbf{r}}=\mathbf{U}_{\mathbf{r}} \mathbf{V}_{\mathbf{r}}{ }^{-1}$.

In this paper we focus on the efficient and numerically accurate computation of low order controllers by using these procedures to solve the frequency-weighted coprime factorization based controller reduction problems formulated in [5]. Here, the following stability enforcing one-sided weights are used: for the left coprime factor reduction the weights are

$$
\widetilde{W}_{o}=I, \quad \widetilde{W}_{i}=\left[\begin{array}{c}
-G \\
I
\end{array}\right](I-K G)^{-1} \widetilde{V}^{-1},
$$

while for the right coprime factor reduction problem the weights are

$$
W_{o}=V^{-1}(I-G K)^{-1}[-G I], \quad W_{i}=I .
$$

The weights are stable TFMs with realizations of order $n+n_{c}$. It can be shown (see for example [13]) that with the above weights, the stability of the closed-loop system is guaranteed if $\left\|\left[\widetilde{U}-\widetilde{U}_{r} \widetilde{V}-\widetilde{V}_{r}\right] \widetilde{W}_{i}\right\|_{\infty}<1$ or $\left\|W_{o}\left[\begin{array}{l}U-U_{r} \\ V-V_{r}\end{array}\right]\right\|_{\infty}<1$. These results justify the frequency-weihted coprime factor controller reduction methods introduced in [5] for the reduction of statefeedback and observer-based controllers. The case of arbitrary stabilizing controllers has been considered in [13]. Both cases are addressed in this paper.

The main computational burden in applying either the FWLCFR or FWRCFR procedure to these problems is the computation of the grammians at Step 1. Apparently, the computation of grammians involves the solutions of at least one Lyapunov equation of order $n+2 n_{c}$. By cleverly exploiting the problem structure, it was shown in [5], that for a strictly proper continuous-time system (i.e., $D=0$ ) and a statefeedback and full-order estimator based controller, it is possible to compute the grammians needed for the frequency-weighted coprime factor reduction by solving only Lyapunov equations of order $n_{c}=n$. In this paper we show that for the general formulation of [13], the frequency-weighted grammians can be computed by solving Lyapunov equations of order $n+n_{c}$. We also extend the results of [5] to the case of nonzero $D$ 
for both continuous- and discrete-time systems. Complete formulas are given for both LCF and RCF based approaches.

In a separate section, we discuss the direct computation of the Cholesky factors of the frequency-weighted grammians. This is a prerequisite for the applicability of the balancing-free square-root accuracy-enhancing techniques to coprime factor controller reduction of [10], along the lines of the model reduction methods developed for the BT in [9] and SPA in [8].

\section{Efficient solution of frequency-weighted controller reduction problems}

\subsection{LCF controller reduction}

We consider the efficient computation of the frequencyweighted controllability grammian at Step 1 of the FWLCFR Procedure for the weights defined in (3). Let $L_{c}$ be any matrix such that $A_{c}+L_{c} C_{c}$ is stable (i.e., the eigenvalues of $A_{c}+L_{c} C_{c}$ lie in the open left half plane for a continuous-time system or in the interior of the unit circle for a discrete-time system). Then, a LCF of $K=\widetilde{V}^{-1} \widetilde{U}$ is given by

$$
[\widetilde{\mathbf{U}} \widetilde{\mathbf{V}}]=\left[\begin{array}{c|cc}
A_{c}+L_{c} C_{c} & B_{c}+L_{c} D_{c} & L_{c} \\
\hline C_{c} & D_{c} & I
\end{array}\right]
$$

The input weighting $\widetilde{W}_{i}$ is a stable TFM having a state space realization $\widetilde{\mathbf{W}}_{\mathbf{i}}:=\left(A_{i}, B_{i}, C_{i}, D_{i}\right)$ of order $n+n_{c}$ [13, see p.503], where

$$
\begin{gathered}
A_{i}=\left[\begin{array}{cc}
A+B \widetilde{R}^{-1} D_{c} C & -B \widetilde{R}^{-1} C_{c} \\
-B_{c} R^{-1} C & A_{c}+B_{c} D \widetilde{R}^{-1} C_{c}
\end{array}\right] \\
B_{i}=\left[\begin{array}{c}
-B \widetilde{R}^{-1} \\
B_{c} D \widetilde{R}^{-1}-L_{c}
\end{array}\right], \quad D_{i}=\left[\begin{array}{c}
-D \widetilde{R}^{-1} \\
\widetilde{R}^{-1}
\end{array}\right] \\
C_{i}=\left[\begin{array}{cc}
R^{-1} C & -D \widetilde{R}^{-1} C_{c} \\
-\widetilde{R}^{-1} D_{c} C & \widetilde{R}^{-1} C_{c}
\end{array}\right]
\end{gathered}
$$

with $R:=I-D D_{c}$ and $\widetilde{R}=I-D_{c} D$.

Construct $[\widetilde{\mathbf{U}} \widetilde{\mathbf{V}}] \widetilde{\mathbf{W}}_{\mathbf{i}}:=\left(\bar{A}_{i}, \bar{B}_{i}, \bar{C}_{i}, \bar{D}_{i}\right)$ with

$$
\begin{gathered}
\bar{A}_{i}=\left[\begin{array}{c|c}
A_{c}+L_{c} C_{c} & B_{c} R^{-1} C L_{c} C_{c}-B_{c} D \widetilde{R}^{-1} C_{c} \\
\hline 0 & A_{i}
\end{array}\right] \\
\bar{B}_{i}=\left[\begin{array}{c}
L_{c}-B_{c} D \widetilde{R}^{-1} \\
B_{i}
\end{array}\right]
\end{gathered}
$$

and let $\bar{P}_{i}$ and $Q$ be the controllability grammian of $[\widetilde{\mathbf{U}} \widetilde{\mathbf{V}}] \widetilde{\mathbf{W}}_{\mathbf{i}}$ and the observability grammian of $[\widetilde{\mathbf{U}} \widetilde{\mathbf{V}}]$, respectively. According to the system type, continuoustime (c) or discrete-time (d), $\bar{P}_{i}$ and $Q$ satisfy the cor- responding Lyapunov equations
(c) $\begin{cases}\bar{A}_{i} \bar{P}_{i}+\bar{P}_{i} \bar{A}_{i}^{T}+\bar{B}_{i} \bar{B}_{i}^{T} & =0 \\ \left(A_{c}+L_{c} C_{c}\right)^{T} Q+Q\left(A_{c}+L_{c} C_{c}\right)+C_{c}^{T} C_{c} & =0\end{cases}$
(d) $\begin{cases}\bar{A}_{i} \bar{P}_{i} \bar{A}_{i}^{T}+\bar{B}_{i} \bar{B}_{i}^{T} & =\bar{P}_{i} \\ \left(A_{c}+L_{c} C_{c}\right)^{T} Q\left(A_{c}+L_{c} C_{c}\right)+C_{c}^{T} C_{c} & =Q\end{cases}$

Partition $\bar{P}_{i}$ in accordance with the structure of the matrix $\bar{A}_{i}$ in (6)

$$
\bar{P}_{i}=\left[\begin{array}{cc}
\bar{P}_{11} & \bar{P}_{12} \\
\bar{P}_{12}^{T} & P_{i}
\end{array}\right]
$$

such that $\bar{P}_{11}$ is an $n_{c} \times n_{c}$ matrix. The approach proposed by Enns in [2] defines

$$
P_{E}=\bar{P}_{11}
$$

as the frequency-weighted controllability grammian.

It follows that the solution of the controller reduction problem for the special weights defined in (3) involves the solution of a Lyapunov equation of order $n+2 n_{c}$ to determine the frequency-weighted controllability grammian $P_{E}$ and a Lyapunov equation of order $n_{c}$ to compute the observability grammian $Q$. The following theorem shows that it is always possible to solve a Lyapunov equation of order $n+n_{c}$ (instead one of order $n+2 n_{c}$ ) to compute the frequency-weighted controllability grammian for the special weights in (3).

Lemma 3.1 For a given $n$-th order system $\mathbf{G}=$ $(A, B, C, D)$ assume that $\mathbf{K}=\left(A_{c}, B_{c}, C_{c}, D_{c}\right)$ is an $n_{c}$-th order stabilizing controller with $I-D D_{c}$ nonsingular. Then the frequency-weighted controllability grammian for Enns' method [2] applied to the frequency-weighted left coprime factorization based controller reduction problem with weights defined in (3) can be computed by solving a Lyapunov equation of order $n+n_{c}$.

Proof: Consider the transformation matrix $T$

$$
T=\left[\begin{array}{ccc}
I_{n_{c}} & 0 & -I_{n_{c}} \\
0 & I_{n} & 0 \\
0 & 0 & I_{n_{c}}
\end{array}\right]
$$

It is easy to see that the controllability grammian $\widetilde{P}_{i}$ for the transformed pair

$$
\left(\widetilde{A}_{i}, \widetilde{B}_{i}\right):=\left(T^{-1} \bar{A}_{i} T, T^{-1} \bar{B}_{i}\right)
$$

has the form

$$
\widetilde{P}_{i}=\left[\begin{array}{cc}
0 & 0 \\
0 & P_{i}
\end{array}\right]
$$

where $P_{i}$ satisfies the appropriate Lyapunov equation

$$
\begin{array}{ll}
A_{i} P_{i}+P_{i} A_{i}^{T}+B_{i} B_{i}^{T}=0 & (c) \\
A_{i} P_{i} A_{i}^{T}+B_{i} B_{i}^{T}=P_{i} & (d)
\end{array}
$$


With $P_{i}$ partitioned similarly as $A_{i}$ in (5)

$$
P_{i}=\left[\begin{array}{ll}
P_{22} & P_{23} \\
P_{23}^{T} & P_{33}
\end{array}\right]
$$

the grammian in the original coordinate basis results as

$$
\bar{P}_{i}=T \widetilde{P}_{i} T^{T}=\left[\begin{array}{ccc}
P_{33} & -P_{23}^{T} & -P_{33} \\
-P_{23} & P_{22} & P_{23} \\
-P_{33} & P_{23}^{T} & P_{33}
\end{array}\right]
$$

The frequency-weighted controllability grammian according to Enns' method is $P_{E}=P_{33}$, the leading $n_{c} \times n_{c}$ block of $\bar{P}_{i}$. But this is also the trailing $n_{c} \times n_{c}$ block of $P_{i}$ in (10), which can be computed by solving the Lyapunov equation (9) of order $n+n_{c}$.

Significant simplifications arise in the case of a statefeedback and full-order observer based controller

$$
\mathbf{K}=\left[\begin{array}{c|c}
A+B F+L C+L D F & -L \\
\hline F & 0
\end{array}\right]
$$

where it is assumed that $A+B F$ and $A+L C$ are both stable. In this case (see [13]), with $L_{c}=-(B+L D)$ we get

$$
[\tilde{\mathbf{U}} \tilde{\mathbf{V}}]=\left[\begin{array}{c|cc}
A+L C & -L & -(B+L D) \\
\hline F & 0 & I
\end{array}\right]
$$

and the input weighting $\mathbf{W}_{\mathbf{i}}$ has the following state space realization of order $n$ [13, p.503]

$$
\widetilde{\mathbf{W}}_{\mathbf{i}}=\left[\begin{array}{c|c}
A+B F & B \\
\hline-C-D F & -D \\
F & I
\end{array}\right]
$$

The following result is an extension of Lemma 2 of [5] to the case of nonzero feedthrough matrix $D$ and covers both the continuous- as well as the discrete-time case.

Corollary 3.2 For a given $n$-th order system $\mathbf{G}=$ $(A, B, C, D)$ and the observer based controller $\mathbf{K}$ (11), suppose $F$ is a state feedback gain and $L$ is a state estimator gain, such that $A+B F$ and $A+L C$ are stable. Then the frequency-weighted controllability and observability grammians for Enns' method [2] applied to the frequency-weighted left coprime factorization based controller reduction problem with weights defined in (3) can be computed by solving Lyapunov equations of order $n$. For a continuous-time system these equations are

$$
\begin{aligned}
& (A+B F) P+P(A+B F)^{T}+B B^{T}=0 \\
& (A+L C)^{T} Q+Q(A+L C)+F^{T} F=0
\end{aligned}
$$

while for a discrete-time system these equations are

$$
\begin{aligned}
& (A+B F) P(A+B F)^{T}+B B^{T}=P \\
& (A+L C)^{T} Q(A+L C)+F^{T} F=Q
\end{aligned}
$$

Proof: The realization of $[\widetilde{\mathbf{U}} \widetilde{\mathbf{V}}] \widetilde{\mathbf{W}}_{\mathbf{i}}$ is

$$
\left[\begin{array}{c|c}
\bar{A}_{i} & \bar{B}_{i} \\
\hline \bar{C}_{i} & \bar{D}_{i}
\end{array}\right]=\left[\begin{array}{cc|c}
A+L C & L C-B F & -B \\
0 & A+B F & B \\
\hline F & F & I
\end{array}\right]
$$

It is easy to verify that in both continuous-time case as well as in the discrete-time case, the controllability grammian $\bar{P}_{i}$ of $[\widetilde{\mathbf{U}} \widetilde{\mathbf{V}}] \widetilde{\mathbf{W}}_{\mathbf{i}}$ can be expressed as

$$
\bar{P}_{i}=\left[\begin{array}{rr}
P & -P \\
-P & P
\end{array}\right]
$$

where $P$ satisfies (12) for a continuous-time system and (13) for a discrete-time system.

\subsection{RCF controller reduction}

Let $F_{c}$ be any matrix such that $A_{c}+B_{c} F_{c}$ is stable. Then, a RCF of $K=U V^{-1}$ is given by

$$
\left[\begin{array}{c}
\mathbf{U} \\
\mathbf{V}
\end{array}\right]=\left[\begin{array}{c|c}
A_{c}+B_{c} F_{c} & B_{c} \\
\hline C_{c}+D_{c} F_{c} & D_{c} \\
F_{c} & I
\end{array}\right]
$$

The output weighting $\mathbf{W}_{\mathbf{o}}$ is a stable TFM having a state space realization $\mathbf{W}_{\mathbf{o}}=\left(A_{o}, B_{o}, C_{o}, D_{o}\right)$ of order $n+n_{c}[13$, p.503], where

$$
\begin{gathered}
A_{o}=\left[\begin{array}{cc}
A_{c}+B_{c} R^{-1} D C_{c} & -B_{c} R^{-1} C \\
-B \widetilde{R}^{-1} C_{c} & A+B D_{c} R^{-1} C
\end{array}\right] \\
B_{o}=\left[\begin{array}{cc}
B_{c} R^{-1} D & B_{c} R^{-1} \\
-B \widetilde{R}^{-1} & -B D_{c} R^{-1}
\end{array}\right] \\
C_{o}=\left[R^{-1} D C_{c}-F_{c}-R^{-1} C\right] \\
D_{o}=\left[R^{-1}-R^{-1} D\right]
\end{gathered}
$$

Construct $\mathbf{W}_{\mathbf{o}}\left[\begin{array}{c}\mathbf{U} \\ \mathbf{V}\end{array}\right]:=\left(\bar{A}_{o}, \bar{B}_{o}, \bar{C}_{o}, \bar{D}_{o}\right)$ with

$$
\begin{aligned}
& \bar{A}_{o}=\left[\begin{array}{c|c}
A_{o} & B_{c} F_{c}-B_{c} R^{-1} D C_{c} \\
\hline 0 & B \widetilde{R}^{-1} C_{c} \\
\hline 0 & A_{c}+B_{c} F_{c}
\end{array}\right] \\
& \bar{C}_{o}=\left[\begin{array}{ll}
C_{o} & F_{c}-R^{-1} D C_{c}
\end{array}\right]
\end{aligned}
$$

Let $P$ and $\bar{Q}_{o}$ be the controllability and observability grammians of $\left[\begin{array}{l}\mathbf{U} \\ \mathbf{V}\end{array}\right]$ and $\mathbf{W}_{\mathbf{o}}\left[\begin{array}{l}\mathbf{U} \\ \mathbf{V}\end{array}\right]$, respectively. According to the system type, $P$ and $\bar{Q}_{o}$ satisfy the corresponding Lyapunov equations
(c) $\begin{cases}\left(A_{c}+B_{c} F_{c}\right) P+P\left(A_{c}+B_{c} F_{c}\right)^{T}+B_{c} B_{c}^{T} & =0 \\ \bar{A}_{o}^{T} \bar{Q}_{o}+\bar{Q}_{o} \bar{A}_{o}+\bar{C}_{o}^{T} \bar{C}_{o} & =0\end{cases}$
(d) $\begin{cases}\left(A_{c}+B_{c} F_{c}\right) P\left(A_{c}+B_{c} F_{c}\right)^{T}+B_{c} B_{c}^{T} & =P \\ \bar{A}_{o}^{T} \bar{Q}_{o} \bar{A}_{o}+\bar{C}_{o}^{T} \bar{C}_{o} & =\bar{Q}_{o}\end{cases}$ 
Partition $\bar{Q}_{o}$ in accordance with the structure of the matrix $\bar{A}_{o}$ in (16)

$$
\bar{Q}_{o}=\left[\begin{array}{cc}
Q_{o} & \bar{Q}_{12} \\
\bar{Q}_{12}^{T} & \bar{Q}_{22}
\end{array}\right]
$$

where $\bar{Q}_{22}$ is an $n_{c} \times n_{c}$ matrix. The approach proposed by [2] defines

$$
Q_{E}=\bar{Q}_{22}
$$

as the frequency-weighted observability grammian.

It follows that the solution of the controller reduction problem for the special weights defined in (4) involves the solution of a Lyapunov equations of order $n_{c}$ to compute controllability grammian $P$ and of a Lyapunov equation of order $n+2 n_{c}$ to determine the frequencyweighted observability grammian $Q_{E}$. We have a similar result to Lemma 3.1 showing that $Q_{E}$ can be determined efficiently by solving only a reduced order Lyapunov equation.

Lemma 3.3 For a given $n$-th order system $\mathbf{G}=$ $(A, B, C, D)$ assume that $\mathbf{K}=\left(A_{c}, B_{c}, C_{c}, D_{c}\right)$ is an $n_{c}$-th order stabilizing controller with $I-D D_{c}$ nonsingular. Then the frequency-weighted observability grammian for Enns' method [2] applied to the frequencyweighted right coprime factorization based controller reduction problem with weights defined in (4) can be computed by solving a Lyapunov equation of order $n+n_{c}$.

Proof: Consider the transformation matrix $T$

$$
T=\left[\begin{array}{ccc}
I_{n_{c}} & 0 & I_{n_{c}} \\
0 & I_{n} & 0 \\
0 & 0 & I_{n_{c}}
\end{array}\right]
$$

It is easy to see that the observability grammian $\widetilde{Q}_{o}$ for the transformed pair

$$
\left(\widetilde{A}_{o}, \widetilde{C}_{o}\right):=\left(T^{-1} \bar{A}_{o} T, \bar{C}_{o} T\right)
$$

has the form

$$
\widetilde{Q}_{o}=\left[\begin{array}{cc}
Q_{o} & 0 \\
0 & 0
\end{array}\right]
$$

where $Q_{o}$ satisfies the appropriate Lyapunov equation

$$
\begin{array}{ll}
A_{o}^{T} Q_{o}+Q_{o} A_{o}+C_{o}^{T} C_{o}=0 & (c) \\
A_{o}^{T} Q_{o} A_{o}+C_{o}^{T} C_{o}=Q_{o} & (d)
\end{array}
$$

With $Q_{o}$ partitioned in accordance with the structure of $A_{o}$ in (15)

$$
Q_{o}=\left[\begin{array}{ll}
Q_{11} & Q_{12} \\
Q_{12}^{T} & Q_{22}
\end{array}\right]
$$

the grammian in original coordinates results as

$$
\bar{Q}_{o}=T^{-T} \widetilde{Q}_{o} T^{-1}=\left[\begin{array}{ccc}
Q_{11} & Q_{12} & -Q_{11} \\
Q_{12}^{T} & Q_{22} & -Q_{12}^{T} \\
-Q_{11} & -Q_{12} & Q_{11}
\end{array}\right]
$$

According to Enns' method, the frequency-weighted observability grammian is $Q_{E}=Q_{11}$, the trailing $n_{c} \times n_{c}$ block of $\bar{Q}_{o}$ in (17). But this is also the leading $n_{c} \times n_{c}$ block of $Q_{o}$ in (20), which can be computed by solving the Lyapunov equation (19) of order $n+n_{c}$.

In the case of a state-feedback and full-order observer based controller (11), we obtain a significant reduction of computational costs. In this case, with $F_{c}=-(C+$ $D F$ ) we get (see [13])

$$
\left[\begin{array}{l}
\mathbf{U} \\
\mathbf{V}
\end{array}\right]=\left[\begin{array}{c|c}
A+B F & -L \\
\hline F & 0 \\
C+D F & I
\end{array}\right]
$$

and the output weighting $\mathbf{W}_{\mathbf{o}}$ has the following state space realization of order $n[13$, p.503]

$$
\mathbf{W}_{\mathbf{o}}=\left[\begin{array}{c|cc}
A+L C & -B-L D & L \\
\hline C & -D & I
\end{array}\right]
$$

The following is a dual result to Lemma 2 of [5] to the case of nonzero feedthrough matrix $D$ and covers also the discrete-time case.

Corollary 3.4 For a given $n$-th order system $\mathbf{G}=$ $(A, B, C, D)$ and the observer based controller $\mathbf{K}$ (11), suppose $F$ is a state feedback gain and $L$ is a state estimator gain, such that $A+B F$ and $A+L C$ are stable. Then the frequency-weighted controllability and observability grammians for Enns' method [2] applied to frequency-weighted right coprime factorization based controller reduction problem with weights defined in (4) can be computed by solving Lyapunov equations of order n. For a continuous-time system these equations are

$$
\begin{aligned}
& (A+B F) P+P(A+B F)^{T}+L L^{T}=0 \\
& (A+L C)^{T} Q+Q(A+L C)+C^{T} C=0
\end{aligned}
$$

while for a discrete-time system these equations are

$$
\begin{aligned}
& (A+B F) P(A+B F)^{T}+L L^{T}=P \\
& (A+L C)^{T} Q(A+L C)+C^{T} C=Q
\end{aligned}
$$

Proof: The realization of $\mathbf{W}_{\mathbf{o}}\left[\begin{array}{l}\mathbf{U} \\ \mathbf{V}\end{array}\right]$ is

$$
\left[\begin{array}{c|c}
\bar{A}_{o} & \bar{B}_{o} \\
\hline \bar{C}_{o} & \bar{D}_{o}
\end{array}\right]=\left[\begin{array}{cc|c}
A+L C & L C-B F & L \\
0 & A+B F & -L \\
\hline C & C & I
\end{array}\right]
$$

It is easy to verify that in both continuous-time as well as in the discrete-time, the observability grammian $\bar{Q}_{o}$ of $\mathbf{W}_{\mathbf{o}}\left[\begin{array}{l}\mathbf{U} \\ \mathbf{V}\end{array}\right]$ can be expressed as

$$
\bar{Q}_{o}=\left[\begin{array}{ll}
Q & Q \\
Q & Q
\end{array}\right]
$$

where $Q$ satisfies (22) for a continuous-time system and (23) for a discrete-time system. 


\section{Square-root techniques}

Accuracy enhancing balancing-free square-root techniques for coprime factor model reduction have been proposed in [10] along the lines of similar methods developed for the BT in [9] and SPA in [8]. In the case of Procedure FWLCFR, the key computation is the determination of the Cholesky factors of the grammians in the form $P_{E}=S_{E} S_{E}^{T}$ and $Q=R^{T} R$, where $Q$ is the standard observability grammian. Having these factors, the reduction of coprime factors at Step 2 can be performed by computing two truncation matrices $L$ and $T$ using the singular value decomposition

$$
R S_{E}=\left[\begin{array}{ll}
U_{1} & U_{2}
\end{array}\right] \operatorname{diag}\left(\Sigma_{1}, \Sigma_{2}\right)\left[\begin{array}{ll}
V_{1} & V_{2}
\end{array}\right]^{T}
$$

with $\Sigma_{1}=\operatorname{diag}\left(\sigma_{1}, \ldots, \sigma_{r_{c}}\right), \Sigma_{2}=\operatorname{diag}\left(\sigma_{r_{c}+1}, \ldots, \sigma_{n_{c}}\right)$ and $\sigma_{1} \geq \ldots \geq \sigma_{r_{c}}>\sigma_{r_{c}+1} \geq \ldots \geq \sigma_{n_{c}} \geq 0$. The square-root method determines $L$ and $T$ as [7]

$$
L=\Sigma_{1}^{-1 / 2} U_{1}^{T} R, \quad T=S_{E} V_{1} \Sigma_{1}^{-1 / 2} .
$$

If the original system is highly unbalanced, potential accuracy losses can be induced in the reduced model if either $L$ or $T$ is ill-conditioned (i.e., nearly rank deficient). To avoid ill-conditioned truncation matrices, balancing-free approaches can be used, as for example, the balancing-free square-root algorithm for the BT introduced by [9]. Similar formulas have been developed for the SPA approach in [8].

The method of Hammarling [3] can be generally employed to solve (9) directly for the Cholesky factor $S_{i}$ of $P_{i}=S_{i} S_{i}^{T}$. By partitioning $S_{i}$ in the form

$$
S_{i}=\left[\begin{array}{cc}
S_{22} & S_{23} \\
0 & S_{33}
\end{array}\right]
$$

with $S_{33} n_{c} \times n_{c}$, the Cholesky factor of the trailing block $P_{33}$ in (10) is $S_{E}=S_{33}$.

In the case of the Procedure FWRCFR, (19) can be solved directly for the Cholesky factor $R_{o}$ of $Q_{o}=$ $R_{o}^{T} R_{o}$. By partitioning $R_{o}$ in the form

$$
R_{o}=\left[\begin{array}{cc}
R_{11} & R_{12} \\
0 & R_{22}
\end{array}\right]
$$

with $R_{11} n_{c} \times n_{c}$, the Cholesky factor $R_{E}$ of the leading block $Q_{11}$ in (20) is $R_{E}=R_{11}$.

\section{Conclusions}

Efficient and numerically reliable balancing related computational approaches have been proposed for the frequency-weighted coprime factors controller reduction with special frequency weights enforcing closedloop stability. By solving lower order Lyapunov equations for computing the grammians, the new procedures are more efficient than the standard frequencyweighted balancing based reduction approach. The frequency weighted grammians can be determined directly in Cholesky factored forms to facilitate the application of square-root and balancing-free accuracy enhancing techniques. For the coprime factor reduction of statefeedback and observer-based controllers, robust numerical software is available in the FORTRAN 77 library SLICOT, together with user friendly interfaces to the computational environments MATLAB and Scilab.

\section{References}

[1] B. D. O. Anderson and Y. Liu. Controller reduction: concepts and approaches. IEEE Trans. Autom. Control, 34:802-812, 1989.

[2] D. Enns. Model Reduction for Control Systems Design. PhD thesis, Dept. Aeronaut. Astronaut., Stanford Univ., Stanford, CA, 1984.

[3] S. J. Hammarling. Numerical solution of the stable, non-negative definite Lyapunov equation. IMA $J$. Numer. Anal., 2:303-323, 1982.

[4] Y. Liu and B. D. O. Anderson. Singular perturbation approximation of balanced systems. Int. J. Control, 50:1379-1405, 1989.

[5] Y. Liu, B. D. O. Anderson, and U. L. Ly. Coprime factorization controller reduction with Bezout identity induced frequency weighting. Automatica, 26:233-249, 1990.

[6] B. C. Moore. Principal component analysis in linear system: controllability, observability and model reduction. IEEE Trans. Autom. Control, 26:17-32, 1981.

[7] M. S. Tombs and I. Postlethwaite. Truncated balanced realization of a stable non-minimal state-space system. Int. J. Control, 46:1319-1330, 1987.

[8] A. Varga. Balancing-free square-root algorithm for computing singular perturbation approximations. Proc. CDC'91, Brighton, UK, pp. 1062-1065, 1991.

[9] A. Varga. Efficient minimal realization procedure based on balancing. In A. El Moudni, P. Borne, and S. G. Tzafestas (Eds.), Proc. of IMACS/IFAC Symp. on Modelling and Control of Technological Systems, Lille, France, vol. 2, pp. 42-47, 1991.

[10] A. Varga. Coprime factors model reduction based on square-root balancing-free techniques. In A. Sydow (Ed.), Computational System Analysis 1992, Proc. 4th Int. Symp. Systems Analysis and Simulation, Berlin, Germany, pp. 91-96, Elsevier, Amsterdam, 1992.

[11] A. Varga and B. D. O. Anderson. Frequencyweighted balancing related controller reduction. Proc. of IFAC'2002 Congress, Barcelona, Spain, 2002.

[12] K. Zhou and J. Chen. Performance bounds for coprime factor controller reductions. Systems \& Control Lett., 26:119-127, 1995.

[13] K. Zhou, J. C. Doyle, and K. Glover. Robust and Optimal Control. Prentice Hall, 1996. 